\title{
A NOTE ON APPROXIMATION BY RATIONAL FUNCTIONS
}

\author{
H. KOBER
}

The theory of the approximation by rational functions on point sets $E$ of the $z$-plane $(z=x+i y)$ has been summarized by J. L. Walsh ${ }^{1}$ who himself has proved a great number of important theorems some of which are fundamental. The results concern both the case when $E$ is bounded and when $E$ extends to infinity.

In the present note a $L_{p}$-theory $(0<p<\infty)$ will be given for the following point sets extending to infinity:

A. The real axis $-\infty<x<\infty, y=0$.

B. The half-plane $-\infty<x<\infty, 0<y<\infty$.

The only poles of the approximating functions are to lie at preassigned points whose number will be required to be as small as possible. ${ }^{2}$ We shall make use of the theory of the class $\mathfrak{S}_{p}$ the fundamental results of which are due to E. Hille and J. D. Tamarkin; $\mathfrak{S}_{p}$ is the set of functions $F(z)$ which, for $0<y<\infty$, are regular and satisfy the inequality

$$
\int_{-\infty}^{\infty}|F(x+i y)|^{p} d x \leqq M^{p} \quad \text { or } \quad|F(z)| \leqq M
$$

for $0<p<\infty$ or $p=\infty$, respectively, where $M$ depends on $F$ and $p$ only. By $|f(x+i y)|_{p}$ we denote

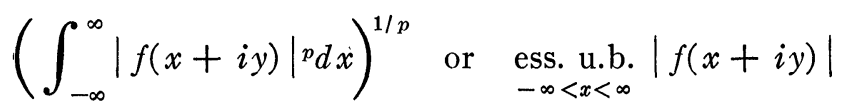

for $0<p<\infty$ or $p=\infty$, respectively, and by $\alpha$ and $\beta$ two arbitrarily fixed points in the upper or lower half-plane, respectively. We obtain the following results: 4

ThEOREM 1. Let $0<p<\infty$ and $F(t) \in L_{p}(-\infty, \infty)$, let $c$ be an integer greater than $p^{-1}$ and $r_{k}(z)=(\alpha-z)^{k}(z-\beta)^{-c-k}[k=0, \pm 1, \pm 2, \cdots]$.

Received by the editors June 26, 1942 .

${ }^{1}$ Interpolation and approximation by rational functions in the complex domain, Amer. Math. Soc. Colloquium Publications, vol. 20, 1935.

${ }^{2}$ Compare Walsh, loc. cit., for example, approximation by polynomials.

${ }^{3}$ Fund. Math. vol. 25 (1935) pp. 329-352, $1 \leqq p<\infty$. For $0<p<1$ see T. Kawata, Jap. J. Math. vol. 13 (1936) pp. 421-430.

${ }_{4}^{4}$ The case $p=\infty$ of each of the results is a special case of Theorem 16, J. L. Walsh, chap. 2. 
Then there are finite linear combinations $s_{n}(z)$ of the $r_{k}(z)$ such that

$$
\left|F(t)-s_{n}(t)\right|_{p}=\left(\int_{-\infty}^{\infty}\left|F(t)-s_{n}(t)\right|^{p} d t\right)^{1 / p} \rightarrow 0 \quad \text { as } n \rightarrow \infty .
$$

Theorem 2. (a) Let $0<p<\infty$ and $F(t) \in L_{p}(-\infty, \infty)$. A necessary and sufficient condition for the existence of rational functions $s_{n}(z)$ such that their only poles lie in a single point of the lower half-plane and that $\left|F(t)-s_{n}(t)\right|_{p \rightarrow 0}$ as $n \rightarrow \infty$ is that $F(t)$ is equivalent to the limit-function of an element $F(z)$ of $\mathfrak{S}_{p}$.

(b) When the latter condition is satisfied then there are rational functions $s_{n}(z)$, with their only poles at $z=\beta$, such that, uniformly in the half-plane $0<y<\infty$,

$$
\left|F(x+i y)-s_{n}(x+i y)\right|_{p} \rightarrow 0 \quad \text { as } n \rightarrow \infty .
$$

By a well known result ${ }^{5}$ concerning $\mathfrak{S}_{p}, 2(\mathrm{~b})$ is a consequence of 2 (a).

We start with giving explicit approximating functions in some special cases of problem (A), taking $\beta=\bar{\alpha}$.

TheOREM 1'. Let $F(t) \in L_{1}(-\infty, \infty)$ or $F(t) \in L_{2}(-\infty, \infty)$, or let $F(t)$ be continuous everywhere, including infinity. ${ }^{6}$ Let $c=2,1,0$ for $p=1,2, \infty$, respectively, and let

$$
s_{n}(z)=\sum_{k=-n}^{n} a_{k} \frac{(\alpha-z)^{k}}{(z-\bar{\alpha})^{k+c}}, \quad a_{k}=\frac{i(\alpha-\bar{\alpha})}{2 \pi} \int_{-\infty}^{\infty} F(t) \frac{(t-\bar{\alpha})^{k+c-1}}{(\alpha-t)^{k+1}} d t .
$$

Then

$$
\begin{aligned}
& \left|F(t)-\frac{1}{N+1} \sum_{n=0}^{N} s_{n}(t)\right|_{1} \text { or }\left|F(t)-s_{N}(t)\right|_{2} \quad \text { or } \\
& \left|F(t)-\frac{1}{N+1} \sum_{n=0}^{N} s_{n}(t)\right|=\underset{\infty}{\text { u.b. }}\left|F(t)-\frac{1}{N+1} \sum_{n=0}^{N} s_{n}(t)\right| \text {, }
\end{aligned}
$$

respectively, tends to zero as $N \rightarrow \infty$. When $F(t)$ is continuous everywhere, including infinity, and of bounded variation in $(-\infty, \infty)$ then the $s_{n}(t)$ converge to $F(t)$ uniformly in $(-\infty, \infty)$.

It will suffice to take $\alpha=i$, the general case being deduced from this one by the substitution $t=\Re(\alpha)+t^{\prime} \Im(\alpha)$. Let $F(t) \in L_{2}(-\infty, \infty)$, $t=\tan (1 / 2) \vartheta \quad[-\pi \leqq \vartheta \leqq \pi]$, and $f(\vartheta)=2\left(1+e^{i \vartheta}\right)^{-1} F(\tan \vartheta / 2)$. Then $F(t) \in L_{2}(-\infty, \infty)$ implies that $f(\vartheta) \in L_{2}(-\pi, \pi)$, and vice versa. Now

${ }^{5}$ Since $s_{n}(t) \in L_{p}$, we have $s_{n}(z) \in \mathfrak{S}_{p}, F(z)-s_{n}(z) \in \mathfrak{S}_{p}$, and we can apply the HilleTamarkin Theorem 2.1 (iii), part 2 , loc. cit.

${ }^{6} \mathrm{~A}$ function $F(t)$ is said to be continuous at infinity wher its limits, as $t \rightarrow \pm \infty$, both exist and are finite and equal. 
the Fourier series $\sum b_{n} e^{i n \vartheta}$, belonging to $f(\vartheta)$, converges to $f(\vartheta)$ in the mean square over $(-\pi, \pi)$. We have $e^{i \vartheta}=(i-t)(i+t)^{-1},(1 / 2)\left(1+e^{i \vartheta}\right)$ $=i(i+t)^{-1}$; taking $a_{n}=i b_{n}$, we arrive finally at the required result. In a similar way we prove the remaining assertions of the theorem. We note ${ }^{7}$ that the sequence $\left\{(2 i \pi)^{-1 / 2}(\alpha-\bar{\alpha})^{1 / 2}(\alpha-t)^{n}(t-\bar{\alpha})^{-n-1}\right\}$ $[n=0, \pm 1, \pm 2, \cdots]$ is a complete orthogonal and normal system with respect to $L_{p}(-\infty, \infty)[1<p<\infty]$.

To prove Theorem 1 , we have to show that, given $\epsilon>0$, there is a finite linear combination $s_{n}(z)$ of the $r_{k}(z)$ such that $\left|F(t)-s_{n}(t)\right|_{p}<\epsilon$. We can find a positive number $b$ and a function $f(t)$ such that $f(t)$ is zero for $|t| \geqq b$ and continuous for $-b \leqq t \leqq b$, and that

$$
\int_{-\infty}^{\infty}|F(t)-f(t)|^{p} d t \leqq \delta, \quad \delta= \begin{cases}(\epsilon / 2)^{p} & \text { for } p>1 \\ (1 / 2) \epsilon^{p} & \text { for } p \leqq 1\end{cases}
$$

The function $g(t)=(t-\beta) c f(t)$ is continuous everywhere, including infinity. From results of $\mathrm{Walsh}^{8}$ we deduce the existence of functions

$$
\sigma_{n}(z)=\sum_{k=-n}^{n} a_{k, n}\left(\frac{\alpha-z}{z-\beta}\right)^{k}, \quad n=0,1,2, \cdots,
$$

$\left|g(t)-\sigma_{n}(t)\right|_{\infty} \rightarrow 0$ as $n \rightarrow \infty$. Taking $s_{n}(z)=(z-\beta)^{-c} \sigma_{n}(z)$, we have

$$
\left|f(t)-s_{n}(t)\right|_{p}^{p}=\left|\frac{g(t)-\sigma_{n}(t)}{(t-\beta)^{c}}\right|_{p}^{p} \leqq\left|g(t)-\sigma_{n}(t)\right|_{\infty}^{p} \int_{-\infty}^{\infty} \frac{d t}{|t-\beta|^{c p}} .
$$

The right side tends to zero as $n \rightarrow \infty$. Therefore, for some $n$, we have $\left|f(t)-s_{n}(t)\right|_{p}^{p}<\delta,\left|F(t)-s_{n}(t)\right|_{p}^{p}<\epsilon^{p}$ which completes the proof.

To prove Theorem $2(\mathrm{a})$, we need some lemmas.

LEMMA 1.9 Let $\varphi(w)$ belong to the Riesz class $H_{p}[0<p<\infty]$, that is to say, let $\varphi(w)$ be regular for $|w|<1$ and satisfy the inequality

$$
\left\|\varphi\left(r e^{i \vartheta}\right)\right\|_{p}=\left(\int_{-\pi}^{\pi}\left|\varphi\left(r e^{i \vartheta}\right)\right|^{p} d \vartheta\right)^{1 / p} \leqq M, \quad 0<r<1,
$$

where $M$ is independent of $r .^{10}$ Then there are polynomials $P_{n}(w)$ $[n=1,2, \cdots]$ such that $\left\|\varphi\left(r e^{i \vartheta}\right)-P_{n}\left(r e^{i \vartheta}\right)\right\|_{p} \rightarrow 0$ as $n \rightarrow \infty$, uniformly for $0<r \leqq 1$.

${ }^{7}$ Cf. H. Kober, a forthcoming paper in Quart. J. Math. Oxford Ser. 1943.

${ }^{8}$ Walsh, loc. cit. chap. 2, Theorem 16. It can also be deduced from Theorem 1' of this paper.

${ }^{9}$ For $p=\infty$ the result holds if and only if $\phi\left(e^{i \vartheta}\right)$ is continuous for $-\pi \leqq \vartheta \leqq \pi$. Cf. Walsh, loc. cit., and Trans. Amer. Math. Soc. vol. 26 (1924) pp. 155-170.

${ }^{10}$ F. Riesz, Math. Zeit. vol. 18 (1923) pp. 87-95. 
By well known properties of the class $H_{p}$, it will suffice to take $r=1$. Let $\varphi(w)=\sum a_{n} w^{n}$. Since, for any fixed $R[0<R<1]$ and uniformly with respect to $\vartheta[-\pi \leqq \vartheta \leqq \pi]$, the series $\sum a_{n} R^{n} e^{i n \vartheta}$ converges to $\varphi\left(R e^{i \vartheta}\right)$, the result can be deduced by means of the well known equation $\left\|\varphi\left(e^{i \vartheta}\right)-\varphi\left(r e^{i \vartheta}\right)\right\|_{p} \rightarrow 0[r \rightarrow 1]$.

Lemma 2. Let $w=(i-z)(i+z)^{-1}$. The function $F(z)$ belongs to $\mathfrak{S}_{p}$ if, and only if, the function $(1+w)^{-2 / p} \varphi(w)$ belongs to $H_{p}$, where $\varphi(w)$ $=F(z)$.

Hille and Tamarkin have proved ${ }^{11}$ that the condition $\varphi(w) \in H_{p}$ is necessary. To define the function $(1+w)^{-2 / p}$, we cut the $w$-plane along the negative real axis from $w=-1$ to $w=-\infty$. When $F(z)$ belongs to $\mathfrak{S}_{p}$ then its limit function $F(t) \quad[y \rightarrow 0, x=t]$ belongs to $L_{p}(-\infty, \infty)$, therefore $\left(1+e^{i \vartheta}\right)^{-2 / p} \varphi\left(e^{i \vartheta}\right)$ to $L_{p}(-\pi, \pi)$. Let $\psi(w)=(1+w)^{-2 / p} \varphi(w)$, and $0<q<p / 3$. By Hölder's theorem, we have

$$
\int_{-\pi}^{\pi}\left|\psi\left(r e^{i \vartheta}\right)\right|^{q} d \vartheta=\left(\int_{-\pi}^{\pi}\left|\varphi\left(r e^{\imath \vartheta}\right)\right|^{p}\right)^{q / p}\left(\int_{-\pi}^{\pi} \frac{d \vartheta}{\left|1+r e^{i \vartheta}\right|^{2 q /(p-q)}}\right)^{1-q / p} .
$$

The right side is uniformly bounded for $0<r<1$. Hence $\psi(w) \in H_{q}$; its limit-function $\psi\left(e^{i \vartheta}\right)$, however, belongs to $L_{p}(-\pi, \pi)$; hence ${ }^{12}$ $\psi(w) \in H_{p}$. Conversely, let $\psi(w) \in H_{p}$. From a result due to R. M. Gabriel ${ }^{13}$ we deduce that

$$
\int_{C}|\psi(w)| p|d w| \leqq 2 \int_{-\pi}^{\pi}\left|\psi\left(e^{w}\right)\right|^{p} d \vartheta
$$

where $C$ is any circle strictly interior to the unit circle $\Gamma[|w|=1]$. By Fatou's theorem, this inequality holds when $C$ is a circle touching $\Gamma$ from within at $w=-1$. Finally, by the transformation $w=(i-z)(i+z)^{-1}$, we deduce that $|F(x+i y)|_{p} \leqq 2^{2 / p}\left\|\psi\left(e^{i \vartheta}\right)\right\|_{p}$ $[0<y<\infty]$ which proves the lemma. In a similar way we can show that when $F(z) \in \mathfrak{S}_{p}$ and $F(t) \in L_{q}(-\infty, \infty)\left[0<p_{q}^{p} \leqq \infty\right]$ then $F(z) \in \mathfrak{S}_{q}$.

LemMa 3. Let $0<p \leqq \infty$, let $f_{n}(z) \in \mathfrak{S}_{p}[n=1,2, \cdots]$, and let $f_{n}(t)$ be the limit-function of $f_{n}(z)$. Let $F(t)$ be defined in $(-\infty, \infty)$ and $\left|F(t)-f_{n}(t)\right|_{p} \rightarrow 0$ as $n \rightarrow \infty$. Then $F(t)$ is equivalent to the limit-function of an element $f(\bar{z})$ of $\mathfrak{S}_{p}$.

${ }^{11}$ Loc. cit. Lemma 2.5.

${ }^{12}$ V. Smirnoff, C. R. Acad. Sci. Paris vol. 188 (1929) pp. 131-133. A. Zygmund, Trigonometrical series, Warsaw, 1935, 7.56(iv).

${ }^{13}$ J. London Math. Soc. vol. 5 (1930) pp. 129-131. Cf. Hille-Tamarkin, Lemmas 2.1 and 2.5 . 
The proof for $0<p<\infty$ is entirely different from that for $1 \leqq p \leqq \infty$, given in a former paper. ${ }^{14}$ Let $0<p<1$ and $\rho>0$, and let $\phi(z) \in \mathfrak{S}_{p}$. Then, for $\rho \leqq y<\infty$, we have $|\phi(z)| \leqq((1 / 2) \pi \rho)^{-1 / p}|\phi(t)|_{p}{ }^{15}$ Since $\left|f_{n}(t)-f_{m}(t)\right|_{p \rightarrow 0}[m>n \rightarrow \infty]$, taking $\phi(z)=f_{m}(z)-f_{n}(z)$ we can deduce that the sequence $\left\{f_{n}(z)\right\}$ converges to an analytic function $f(z)$, uniformly for $-\infty<x<\infty, \rho<y<\infty$. Since there is a constant $K$, independent of $n$, such that $\left|f_{n}(t)\right|_{p} \leqq K$, we have $\left|f_{n}(x+i y)\right|_{p} \leqq K$, and we can deduce that $|f(x+i y)|_{p} \leqq K$ for any positive $y$. Hence $f(z) \in \mathfrak{S}_{p}$. We are left to show that $f(t)$, the limit-function of $f(z)$, is equivalent to $F(t)$ in $(-\infty, \infty)$. Given $\epsilon>0$, we have $\mid\left(f_{m}(x)-\left.f_{N}(x)\right|_{p} ^{p}<\epsilon / 12\right.$ for $m \geqq N$, fixing $N$ in a suitable way, and $\left|f_{N}(x+i y)-f_{N}(x)\right|_{p}^{p} \leqq \epsilon / 6$ for $0<y \leqq \delta=\delta(\epsilon, N)$. Hence

$$
\begin{aligned}
& \left|f_{m}(x+i y)-f_{m}(x)\right|_{p}^{p} \leqq\left|f_{m}(x+i y)-f_{N}(x+i y)\right|_{p}^{p} \\
& +\left|f_{m}(x)-f_{N}(x)\right|_{p}^{p}+\left|f_{N}(x+i y)-f_{N}(x)\right|_{p}^{p} \leqq \epsilon / 3
\end{aligned}
$$

for $m \geqq N, 0<y \leqq \delta$, since the first term on the right side is not greater than the second term. Given $M>0$, we have

$$
\begin{aligned}
\int_{-M}^{M}\left|f(x)-f_{m}(x)\right|{ }^{p} d x & \leqq \int_{-M}^{M}\left|f(x+i y)-f_{m}(x+i y)\right|{ }^{p} d x \\
& +|f(x+i y)-f(x)|_{p}^{p}+\left|f_{m}(x+i y)-f_{m}(x)\right|_{p}^{p} .
\end{aligned}
$$

The right side is smaller than $\epsilon$ for $m \geqq m_{0}(\epsilon)$, as we see fixing a suitable value for $y$. Consequently $f(x)=F(x)$ almost everywhere in any finite interval $(-M, M)$ and, therefore, in $(-\infty, \infty)$. With a slight alteration, the proof holds for $1 \leqq p<\infty$.

By the lemma, the necessity of the condition in Theorem 2(a) is evident. For $s_{n}(t)$ belongs to $L_{p}(-\infty, \infty)$, therefore $s_{n}(z)$ to $\mathfrak{S}_{p}$. To prove its sufficiency, we take first $1<p<\infty$. By Theorem 1 , there are rational functions $R_{n}(z)$ such that their only poles lie at $z=\bar{\beta}$ and $z=\beta$ and that $\left|F(t)-R_{n}(t)\right|_{p} \rightarrow 0$ as $n \rightarrow \infty$. Taking $R_{n}(z)=s_{n}(z)+\sigma_{n}(z)$, where the rational functions $\dot{s}_{n}$ and $\sigma_{n}$ vanish at infinity and have no poles other than at $z=\bar{\beta}$ or $z=\beta$, respectively, we have $s_{n}(z) \in \mathfrak{S}_{p}$, $\overline{\sigma_{n}(\bar{z})} \in \mathfrak{S}_{p}$. Denoting by $\mathfrak{S} f$ the Hilbert operator

$$
\mathfrak{S} f=\frac{1}{\pi} P V \int_{-\infty}^{\infty} \frac{f(t) d t}{t-x}
$$

we have $|\mathfrak{S} f|_{p} \leqq C_{p}|f|_{p}, \mathfrak{S} F=i F(x)$ and $\mathfrak{S} s_{n}=i s_{n}(x), \mathfrak{S} \sigma_{n}=-i \sigma_{n}(x) .{ }^{14}$

${ }^{14}$ H. Kober, Bull. Amer. Math. Soc. vol. 48 (1942) pp. 421-427.

${ }^{15}$ This can be shown by means of the inequality (73), M. Plancherel and G. Polya, Comment. Math. Helv. vol. 10 (1937-1938) pp. 110-163. 
Hence

$$
\begin{aligned}
2\left|F(t)-s_{n}(t)\right|_{p} & =\left|i F+\mathfrak{S} F-\left(i R_{n}+\mathfrak{S} R_{n}\right)\right|_{p} \\
& \leqq\left|F-R_{n}\right|_{p}+\left|\mathfrak{S}\left(F-R_{n}\right)\right|_{p} \leqq\left(C_{p}+1\right)\left|F-R_{n}\right|_{p}
\end{aligned}
$$

which tends to zero as $n \rightarrow \infty$. Hence $\left|F(t)-s_{n}(t)\right|_{p} \rightarrow 0$ as $n \rightarrow \infty$.

Let now $0<p \leqq 1$ and $F(z) \in \mathfrak{S}_{p}$, let $\beta=-i, z=i(1-w)(1+w)^{-1}$ and $\varphi(w)=F(z)$. Given $\epsilon>0$, from the Lemmas 2 and 1 we infer the existence of a polynomial $P(z)$ such that

$$
\int_{-\pi}^{\pi}\left|\varphi\left(e^{i \vartheta}\right)\left(1+e^{i \vartheta}\right)^{-2 / p}-P\left(e^{i \vartheta}\right)\right| p d \vartheta \leqq \epsilon / 4 .
$$

Hence

$$
\int_{-\infty}^{\infty}\left|F(t)-\left(1+e^{i v}\right)^{2 / p} P\left(\frac{i-t}{i+t}\right)\right|^{p} d t \leqq \epsilon / 2,
$$

where $t=\tan \vartheta / 2$. Let $b$ be an integer, $p^{-1}<b \leqq 1+p^{-1}$. Then the rational function $\chi(z)=(2 i)^{b}(i+z)^{-b} P\left\{(i-z)(i+z)^{-1}\right\}$ has no singularity except at $z=-i$. Since $\chi(t) \in L_{p}(-\infty, \infty)$, we have $|\chi(t)|_{p}$ $=C<\infty$. Now the function $\left(1+e^{i \vartheta}\right)^{2 / p-b}$ can be approximated by polynomials $Q_{m}\left(e^{i \vartheta}\right) \quad[m=1,2, \cdots]$, uniformly for $-\pi \leqq \vartheta \leqq \pi$. Hence, for some $m$, we have

$$
\int_{-\infty}^{\infty}\left|\left(1+e^{i \vartheta}\right)^{2 / p} P\left(\frac{i-t}{i+t}\right)-\chi(t) Q_{m}\left(\frac{i-t}{i+t}\right)\right|^{p} d t<\epsilon / 2 .
$$

Thus $\left|F(t)-\chi(t) Q_{m}\left\{(i-t)(i+t)^{-1}\right\}\right|_{p}<\epsilon^{1 / p}$. This completes the proof which, slightly altered, holds for $1<p \leqq 2$.

For $p=1,2, \infty$, we obtain explicit approximating functions by Theorem $1^{\prime}$ and by the lemma:

Let $1 \leqq p \leqq \infty$ and $F(z) \in \mathfrak{S}_{p}$, let $a$ be an integer and $a \geqq 0$ for $p=1$, $a \geqq 2$ for $p=\infty, a \geqq 1$ otherwise; then

$$
\int_{-\infty}^{\infty} F(t) \frac{(\alpha-t)^{n}}{(t-\beta)^{n+a}} d t=0 \text { for } n=0,1,2, \cdots .
$$

THEOREM $2^{\prime}$. Let $p=2,1$, or $\infty$ and $c=1,2$, or 0 , respectively; let $F(z) \in \mathfrak{S}_{p}$ and $F(t)$, the limit-function of $F(z)$, be continuous everywhere including infinity when $p=\infty$. Let $s_{n}(z)$ be defined by

$$
\sum_{k=0}^{n} a_{k} \frac{(\bar{\beta}-z)^{k}}{(z-\beta)^{k+c}}[p=2] \text { or } \frac{1}{n+1} \sum_{j=0}^{n} \sum_{k=0}^{j} a_{k} \frac{(\bar{\beta}-z)^{k}}{(z-\beta)^{k+c}}\left[\begin{array}{l}
p=1, \\
p=\infty
\end{array}\right]
$$


where

$$
a_{k}=i \frac{\bar{\beta}-\beta}{2 \pi} \int_{-\infty}^{\infty} F(t) \frac{(t-\beta)^{k+c-1}}{(\bar{\beta}-t)^{k+1}} d t .
$$

Then, uniformly for $0 \leqq y<\infty,\left|F(x+i y)-s_{n}(x+i y)\right|_{p} \rightarrow 0$ as $n \rightarrow \infty$.

Applying Theorem $2^{\prime}$ to the components of $g(z)=(1 / 2) s(1-s)$ $\Gamma((1 / 2) s) \pi^{-s / 2} \zeta(s),{ }^{16}$ where $\zeta(s)$ is the Riemann zeta-function and $z=i(1-2 s)$, we can deduce the following corollary:

Let $0 \leqq a<\infty, q=i(1-a), r=i(1+a)$, let

$$
\begin{gathered}
\vartheta(x)=\sum_{n=-\infty}^{\infty} e^{-n^{2} \pi x} ; \quad b_{0}=\vartheta(1) / 2+(1-a / 2) \int_{1}^{\infty} v^{a / 4} \vartheta^{\prime}(v) d v \\
L_{n}^{(j)}(x)=\sum_{k=0}^{n} C_{n+j, k+j} \frac{(-x)^{k}}{k !} ; \\
b_{n}=(-1)^{n} \int_{1}^{\infty} v^{a / 4} \vartheta^{\prime}(v)\left\{L_{n}^{(0)}(\log v) / 2-(a / 2) L_{n}^{(-1)}(\log v) / 2\right\} d v
\end{gathered}
$$

Then the series

$$
\sum_{n=0}^{\infty} b_{n}\left\{\left(\frac{q-z}{r+z}\right)^{n}+\left(\frac{q+z}{r-z}\right)^{n}\right\}
$$

converges to $g(z)$ uniformly for $-\infty<x<\infty,-a \leqq y \leqq a$, while it does not converge whenever $|y|>a$.

The series takes a simple form for $a=0$ (critical line).

The UNIVERsity,

Edgbaston, Birmingham, England

\footnotetext{
${ }^{16}$ In fact to the function $g_{1}(z-i a) \in \mathfrak{S}_{\infty}$, where $g(z)=g_{1}(z)+g_{1}(-z), \quad g_{1}(z)$ $=\left(\left(1+z^{2}\right) / 16\right) \int_{1}^{\infty}\{\vartheta(t)-1\} t^{(i z-3) / 4} d t-(1 / 4)-(i z / 4)\{\vartheta(1)-1\}$.
} 